\title{
Factores de Crecimiento, Variables Antropométricas Maternas y Tamaño de Recién Nacidos de Término
}

\author{
CLEMENCIA CABRERA F. ${ }^{1}$, CARMEN SOTO L. ${ }^{1}$, KATIA SEPÚLVEDA A. ${ }^{1}$, \\ MARJORIE CISTERNA C. ${ }^{1}$, HILDA TEUBER L. ${ }^{1}$, SONIA SEPÚLVEDA V. ${ }^{1}$, \\ JORGE CABRERA D. ${ }^{2}$, GERMÁN CRUZ B. ${ }^{3}$, HERIBERTO ARANEDA C. ${ }^{4}$ \\ 1. Matrona, Departamento Obstetricia y Ginecología, Facultad de Medicina, Universidad de Concepción, Chile. \\ 2. Médico-Cirujano, Especialista en Ginecología y Obstetricia, Departamento Obstetricia y Ginecología, \\ Facultad de Medicina, Universidad de Concepción, Chile. \\ 3. Médico-Cirujano, Especialista en Ginecología y Obstetricia, Clínica Francesa, Concepción, Chile. \\ 4. Tecnólogo Médico, PhD, Departamento Obstetricia y Ginecología, Facultad de Medicina, Universidad de Concepción, \\ Chile.
}

\section{ABSTRACT \\ Insulin, Cortisol, IGF, IGFBP-3 and Maternal Anthropometric Variables in Full Term Newborns}

Introduction: Fetal growth is a physiological phenomenon involving maternal, placentary and fetal factors. Objective: To analyze growth factors and maternal anthropometric variables in full-term newborns (NB) and compare them to their size. Patients and Methods: 138 newborns, 37-41 gestational weeks, sorted according to size in low, normal and large for gestational age. Umbilical cord blood was measured for: ELISA, insulin, cortisol, IGF, IGFBP-3. Results: A statistically significant difference was seen between maternal weight at the beginning and end of pregnancy, and BMI(body mass index) at the beginning of pregnancy with the size of the NB $(p<0.001)$. There was no significant difference between growth factors and NB size. Conclusion: Insulin, Cortisol, IGF1, IGFBP-3 do not show statistically significant differences with the size of a newborn, although the mean value of growth factors in normal and large NB for gestational age, were twice than mean values in low for gestational age newborns. There is a positive, statistically significant correlation between the size of the newborn, and the size of the mother, weight at the beginning and end of the pregnancy and the Body Mass Index of the mother at the beginning of the pregnancy.

(Key words: Insulin, cortisol, IGF-1, IGFBP-3, maternal antropometric variables).

Rev Chil Pediatr 2010; 81 (1): 20-27

Trabajo recibido el 23 de enero de 2009, devuelto para corregir el 08 de abril de 2009, segunda versión el 07 de octubre de 2009, aceptado para publicación el 21 de noviembre de 2009.

Proyecto financiado por la Dirección de Investigación de la Universidad de Concepción, № 205.084.011-1.0

Correspondencia a:

Clemencia Cabrera Fajardo

E-mail: ccabrera@udec.cl 


\section{RESUMEN}

Introducción: El crecimiento fetal es un fenómeno fisiológico complejo en el que intervienen factores maternos, placentarios y fetales. Objetivo: Analizar factores de crecimiento y variables antropométricas maternas en recién nacidos (RN) de término y compararlos con el tamaño de los RN. Pacientes y Método: Estudio prospectivo, descriptivo, transversal, que incluyó a $138 \mathrm{RN}$ entre 37 y 41 semanas, clasificados según tamaño en PEG, AEG y GEG. En sangre de cordón se midió por ELISA, Insulina, cortisol, IGF-1 e IGFBP-3. Resultados: Hubo diferencia estadísticamente significativa $(\mathrm{p}<0,01)$ entre: peso materno al inicio, peso materno al término e índice de masa corporal (IMC) al inicio de la gestación con el tamaño del RN. Hubo correlación positiva entre las variables antropométricas maternas y el peso del $\mathrm{RN}(\mathrm{p}<0,001)$. No hubo diferencia significativa entre los factores de crecimiento y el tamaño de los RN. Conclusión: Insulina, cortisol, IGF-1 e IGFBP-3 no muestran diferencias estadísticamente significativas según el tamaño de RN, aunque las medianas de los factores de crecimiento analizados en RN AEG y GEG duplican los valores de los RN PEG. Se observó correlación positiva y estadísticamente significativa entre el tamaño del $\mathrm{RN}$ y talla materna, peso al inicio de la gestación, peso al término de la gestación e IMC al inicio de la gestación.

(Palabras clave: Insulina, cortisol, IGF-1, IGFBP-3, variables antropométricas maternas).

Rev Chil Pediatr 2010; 81 (1): 20-27

\section{Introducción}

El crecimiento es un fenómeno fisiológico complejo, en el cual los seres vivos incrementan su masa, se diferencian y maduran para expresar su potencial ${ }^{1,2}$. Tanto el crecimiento fetal como su ritmo madurativo es el resultado de la interacción de factores maternos, placentarios y fetales, que conducen a la programación del fenotipo $^{3,4}$. En este proceso, las hormonas implicadas en el control del crecimiento fetal son diferentes a las expresadas en el crecimiento postnatal.

Actualmente, se conoce que además de las variables fisiológicas, la regulación del crecimiento fetal depende de la disponibilidad de nutrientes y del eje: glucosa, insulina y factores de crecimiento tipo insulina (IGFs) ${ }^{5}$. La insulina, regula el crecimiento fetal estimulando la síntesis proteica y la expresión de IGF-I, promoviendo el crecimiento fetal y actuando como señal de disponibilidad de nutrientes. Durante el desarrollo fetal, los diferentes tejidos tienen la capacidad de expresar IGF-I y sus proteínas de transporte; las IGFs, son péptidos con estructura similar a la insulina, inducen crecimiento y proliferación celular e influencian el transporte de glucosa y aminoácidos a través de la placenta ${ }^{6-10}$. Por otra parte, los glucocorticoides son esenciales para el desarrollo y maduración de los órganos fetales antes del nacimiento; el cortisol, tiene efecto catabólico y su incremento se asocia con parto prematuro y restricción del crecimiento fetal ${ }^{11,12}$.

Un número creciente de evidencias sugieren que tanto la restricción del crecimiento fetal como la macrosomía se asocian a enfermedades del adulto tales como hipertensión, diabetes tipo II, dislipidemia, desórdenes reproductivos y riesgo de cáncer mamario, prostático y colorectal en la vida adulta ${ }^{13-15}$.

El objetivo de este estudio es analizar y medir en sangre de cordón los niveles de insulina, cortisol, IGF-1 e IGFBP-3 y variables antropométricas maternas, para determinar la asociación entre estos factores, la edad gestacional (EG) y el tamaño del RN. Nuestra hipótesis es que los niveles de Insulina, cortisol, IGF-1, e IGFBP-3 dependen del tamaño del RN de término.

\section{Pacientes y Método}

Estudio prospectivo, descriptivo de corte transversal, realizado desde Junio del 2006 a Junio del 2007, en el Hospital Guillermo Grant Benavente y Clínica Francesa de Concepción.

Se incluyó correlativamente a 138 recién nacidos cuyos partos ocurrieron de Lunes a Viernes y de 08:00 a 20:00 hrs, con edad gestacional pediátrica (EGP) igual o mayor a 37 semanas cuyas madres consintieron participar en el estudio firmando un consentimiento in- 
formado conforme al protocolo aprobado por ambas instituciones. Los RN se clasificaron de acuerdo a la curva de crecimiento fetal, del Ministerio de Salud de Chile del año 2001, en: a) $\mathrm{RN}$ adecuados para la edad gestacional (AEG), con peso al nacer entre el percentil 10 y 90 para la edad gestacional $(n=42)$; b) $\mathrm{RN}$ pequeños para la edad gestacional (PEG), bajo el percentil $10(n=47)$ y c) $R N$ grandes para la edad gestacional (GEG) sobre el percentil $90(n=49)$. Se incluyeron los $R N$ vivos de término sin patología con edad gestacional pediátrica entre 37 y 41 semanas, cuyas madres no presentaron patologías asociadas al embarazo. No se incluyeron los RN con malformaciones congénitas, hijos de madres diabéticas, embarazo múltiple, síndrome hipertensivo del embarazo y tratamiento antenatal con corticoides.

Los datos fueron recolectados en un instrumento especialmente diseñado para este fin. Se entrevistó a las madres en el postparto y para completar los datos se revisaron los registros de las Unidades de Parto y Recién Nacidos, carné perinatal y carné del recién nacido en ambas instituciones.

\section{Obtención de la Muestra}

Seccionado el cordón umbilical se obtuvo 5 cc de sangre de cordón umbilical de la porción adherida a la placenta. Las muestras se recolectaron en tubos Vacutainer, sin anticoagulante y se almacenaron a $4{ }^{\circ} \mathrm{C}$ y se procesaron dentro de 24 horas. Las muestras fueron centrifugadas a $3000 \mathrm{rpm}$ por 15 minutos a temperatura am- biente, el suero obtenido se alicuotó en fracciones de $0,6 \mathrm{ml}$ y fueron congeladas a $-20{ }^{\circ} \mathrm{C}$ para su posterior análisis. Las muestras fueron procesadas en el Laboratorio de Medicina Perinatal del Servicio de Obstetricia y Ginecología del Hospital Clínico Guillermo Grant Benavente de Concepción. Los ensayos se hicieron por método de ELISA, amplificado por quimioluminiscencia, con kits de Siemens, UK en un equipo automatizado IMMULITE-2000, Siemens Medical Solutions Diagnostics, Los Angeles CA, USA. En cada muestra se midió insulina $(\mathrm{mUI} / \mathrm{ml})$, cortisol $(\mathrm{ug} / \mathrm{dL})$, IGF-1 $(\mathrm{ng} /$ $\mathrm{ml})$ e IGFBP-3 $(\mathrm{mg} / \mathrm{l})$.

El análisis de los datos se hizo con el software estadístico SPSS 12.0 Inc. Chicago, Illinois, USA. Se aplicó ANOVA y correlación de Pearson. Se consideró diferencia significativa con un valor de $\mathrm{p}<0,05$.

\section{Resultados}

El peso al inicio de la gestación fue $63,5 \pm$ $8,2,58,2 \pm 11,2$ y $73,2 \pm 12,8 \mathrm{~kg}(\mathrm{p}<0,02)$ en AEG, PEG y GEG, respectivamente, así como el peso al término de la gestación fue 75,2 \pm $8,1,68,5 \pm 9,9$ y $85,3 \pm 11,9 \mathrm{~kg}(\mathrm{p}<0,003)$ y el IMC al inicio de la gestación 25,9 $\pm 3,8,24,2$ $\pm 4,2$ y $29,1 \pm 4,8(\mathrm{p}<0,02)$ en AEG, PEG y GEG, respectivamente, tuvieron diferencias significativas entre los 3 grupos. No hubo diferencias significativas en los grupos de $\mathrm{RN}$ con respecto a la edad, talla y nivel de escolaridad de las gestantes y ganancia de peso durante la gestación (tabla 1).

Tabla 1. Variables demográficas y antropométricas de las gestantes según tamaño del recién nacido

\begin{tabular}{|lcccc|}
\hline & AEG & PEG & GEG & Valor $\mathbf{p}$ \\
\hline Edad (años) & $\mathbf{n = 4 2}$ & $\mathbf{n = 4 7}$ & $\mathbf{n = 4 9}$ & 0,28 \\
\hline Escolaridad (años) & $28,7 \pm 6,04$ & $27,03 \pm 7,1$ & $27,4 \pm 6,6$ & 0,58 \\
\hline Peso inicio embarazo ${ }^{\circ}$ trimestre $(\mathrm{kg})$ & $12,3 \pm 2,6$ & $10,7 \pm 2,9$ & $9,9 \pm 2,8$ & 0,02 \\
\hline Peso término embarazo $(\mathrm{kg})$ & $63,5 \pm 8,2$ & $58,2 \pm 11,2$ & $73,2 \pm 12,8$ & 0,003 \\
\hline Indice de Masa Corporal (IMC) & $75,2 \pm 8,1$ & $68,5 \pm 9,9$ & $85,3 \pm 11,9$ & 0,02 \\
Ganancia peso (kg) & $25,9 \pm 3,8$ & $24,2 \pm 4,2$ & $29,1 \pm 4,8$ & 0,45 \\
\hline Talla $(\mathrm{cm})$ & $11,6 \pm 5,2$ & $10,2 \pm 5,0$ & $11,9 \pm 5,1$ & 0,49 \\
\hline
\end{tabular}

Los datos se presentan en promedio. 
Se observó una correlación positiva entre el peso del $\mathrm{RN}$ y el peso materno al inicio de la gestación $(\mathrm{p}<0,0001)$, talla materna $(\mathrm{p}<0,007)$, peso al término de la gestación $(\mathrm{p}<0,0001)$ e IMC $(\mathrm{p}<0,0001)$ (tabla 2$)$.

No existe correlación entre los factores de crecimiento fetal (insulina, cortisol, IGF-1 e IGFBP-3) y el peso de los RN, clasificados según tamaño en AEG, PEG y GEG (tabla 3).

Los pesos promedios de los recién nacidos, clasificados en AEG, GEG y PEG se muestran en la tabla 4. El peso promedio de los RN de 37 semanas se comparó con el de los nacidos a las 40 semanas encontrando que el peso fue $388 \mathrm{~g}$ superior en los AEG, $490 \mathrm{~g}$ en los PEG y $226 \mathrm{~g}$ en los GEG, respecto del mismo grupo a

Tabla 2. Variables antropométricas maternas y peso del recién nacido

\begin{tabular}{|lcl|}
\hline Variable & $\mathbf{r}^{*}$ & \multicolumn{1}{c|}{$\mathbf{p}$} \\
\hline Peso inicio gestación & 0,508 & 0,0001 \\
\hline Talla materna & 0,228 & 0,007 \\
\hline Peso término gestación & 0,563 & 0,0001 \\
\hline Índice de masa corporal (IMC) & 0,444 & 0,0001 \\
\hline Ganancia de peso & 0,128 & 0,18 \\
\hline
\end{tabular}

*Correlación Pearson. El coeficiente de correlación r de Pearson calculado se docimó a través de la prueba T de Student, que parte de la comprobación de las hipótesis: Ho: Hipótesis nula. $\mathrm{Rho}=0$ versus la $\mathrm{H} 1$. Hipótesis alternativa Rho $>0$. las 37 semanas, lo que equivale a una ganancia de peso de los RN AEG de 12,7\%, en los PEG de $21,32 \%$ y en los GEG de $5,54 \%$ (tabla 5 ).

La figura 1 muestra los valores en mediana de Insulina en RN AEG, PEG y GEG en las distintas edades gestacionales. Se observa en los RN PEG un incremento brusco de la insu-

Tabla 3. Insulina, cortisol, IGF-1 e IGFBP-3 en recién nacidos clasificados según tamaño. AEG, PEG y GEG

\begin{tabular}{|lcc|}
\hline Recién nacidos AEG & r & p \\
Insulina & 0,30 & 0,19 \\
Cortisol & $-0,12$ & 0,59 \\
IGF1 & 0,27 & 0,23 \\
IGFBP3 & $-0,01$ & 0,96 \\
Recién nacidos PEG & $\mathbf{r}$ & $\mathbf{p}$ \\
Insulina & 0,2 & 0,28 \\
Cortisol & 0,1 & 0,70 \\
IGF1 & 0,3 & 0,15 \\
IGFBP3 & 0,4 & 0,07 \\
Recién nacidos GEG & $\mathbf{r}$ & $\mathbf{p}$ \\
Insulina & 0,03 & 0,90 \\
Cortisol & 0,18 & 0,44 \\
IGF1 & $-0,2$ & 0,51 \\
IGFBP3 & 0,02 & 0,92 \\
\hline
\end{tabular}

Correlación de Pearson.

Tabla 4. Peso y talla de los recién nacidos clasificados de acuerdo al tamaño

\begin{tabular}{|lccc|}
\hline & AEG & PEG & GEG \\
\hline Peso RN (gr) & $3317,8 \pm 284,2$ & $2567,4 \pm 232$ & $4251,6 \pm 233,2$ \\
\hline Talla RN (cm) & $50,6 \pm 2,01$ & $47,8 \pm 1,9$ & $53,5 \pm 1,7$ \\
\hline
\end{tabular}

Los datos se presentan en promedio.

Tabla 5. Peso promedio de los recién nacidos de 37 semanas, versus peso promedio de los recién nacidos de $\mathbf{4 0}$ semanas y porcentaje de ganancia

\begin{tabular}{|ccccc|}
\hline & $\begin{array}{c}\text { Peso(g) } \mathbf{3 7} \text { sem } \\
\mathbf{n = 1 7}\end{array}$ & $\begin{array}{c}\text { Peso(g) } \mathbf{4 0} \text { sem } \\
\mathbf{n = 3 1}\end{array}$ & $\begin{array}{c}\text { Ganancia peso } \\
\mathbf{( g )}\end{array}$ & $\begin{array}{c}\text { \% de } \\
\text { ganancia }\end{array}$ \\
\hline AEG & $3063 \quad(n=6)$ & $3451 \quad(n=6)$ & 388 & 12,7 \\
\hline PEG & $2298 \quad(n=8)$ & $2788(n=8)$ & 490 & 21,32 \\
\hline GEG & $4079(n=3)$ & $4305 \quad(n=17)$ & 226 & 5,54 \\
\hline
\end{tabular}

El peso de los recién nacidos se presentan en promedio. 
lina a las 38 semanas para luego descender (NS). En los RN AEG el incremento máximo fue a las 40 semanas, y en los GEG a las 39 semanas para luego descender.

La figura 2 muestra los valores de medianas de cortisol para RN AEG, PEG y GEG a las distintas edades gestaciones. Destaca que el grupo PEG cursa con niveles superiores de cortisol hasta las 39 semanas, para luego igualar los niveles con los otros grupos.

La figura 3, muestra los valores de mediana de IGF-1 y edad gestacional pediátrica en $\mathrm{RN}$ de término AEG, PEG y GEG. En los RN AEG se observa un aumento de los niveles de IGF-1 en la semana 38, para luego descender progresivamente. En cambio los RN PEG, presentan un nivel máximo en la semana 38 , mostrando un descenso en la semana 39. Los RN GEG, alcanzan su máximo nivel en la semana 40 .

Los valores de mediana de IGFBP-3 y edad gestacional pediátrica en $\mathrm{RN}$ de término AEG, PEG y GEG se muestran en la figura 4. Los RN AEG, muestran su máximo nivel de IGFBP-3 en la semana 39, los RN PEG en la semana 40 al igual que los RN GEG (NS).

El peso promedio y edad gestacional pediátrica en $\mathrm{RN}$ de término se muestra en la figura 5. Los RN AEG pesan en promedio de $3063 \mathrm{~g}$ a las $37 \mathrm{sem}$, incrementando hasta la semana 40 a 3451 g (+388 g). Los RN PEG registran un peso promedio de $2298 \mathrm{~g}$ a las $37 \mathrm{sem}$ y 2788 $\mathrm{g}$ a las $40 \mathrm{sem}(+490 \mathrm{~g})$. Los RN GEG pesan $4079 \mathrm{~g}$ en promedio a las 37 sem y $4305(+226 \mathrm{~g}) \mathrm{g}$ a las $40 \mathrm{sem}$.

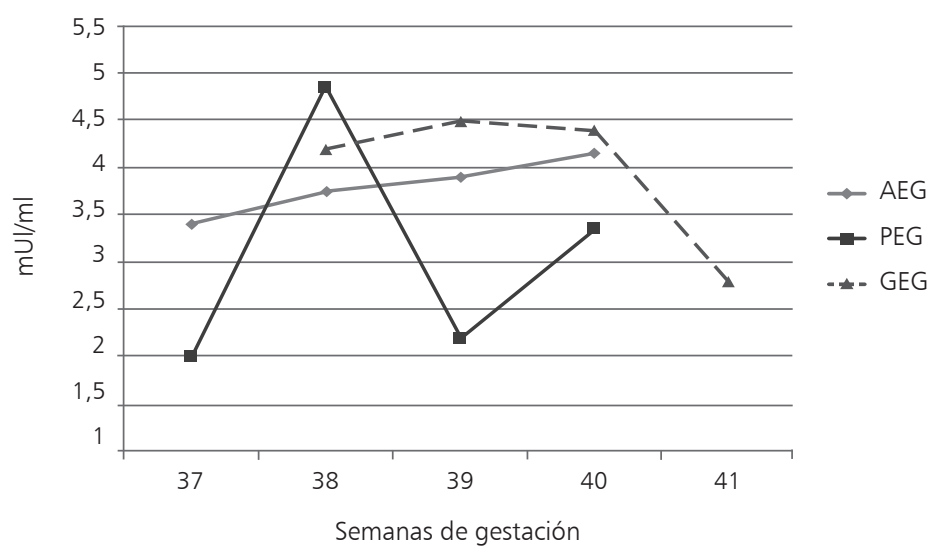

Figura 1. Valores en mediana de Insulina y edad gestacional pediátrica en RN de término.

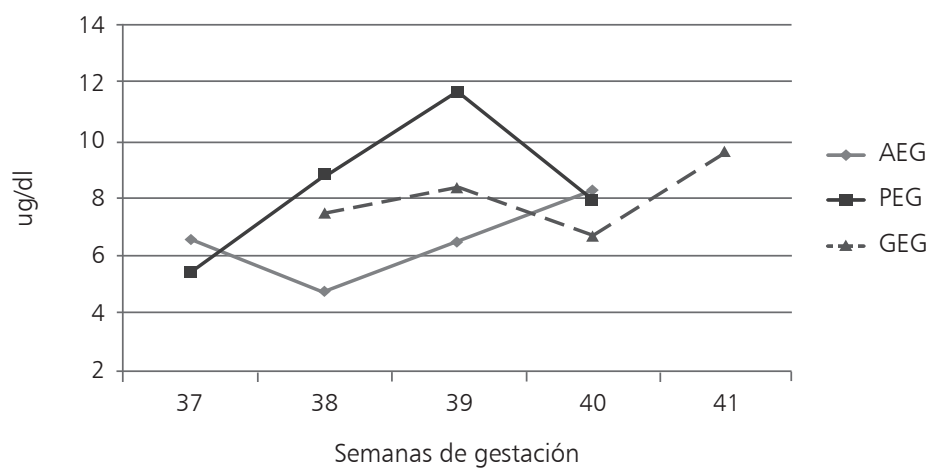

Figura 2. Valores de mediana de cortisol y edad gestacional pediátrica en RN de término.

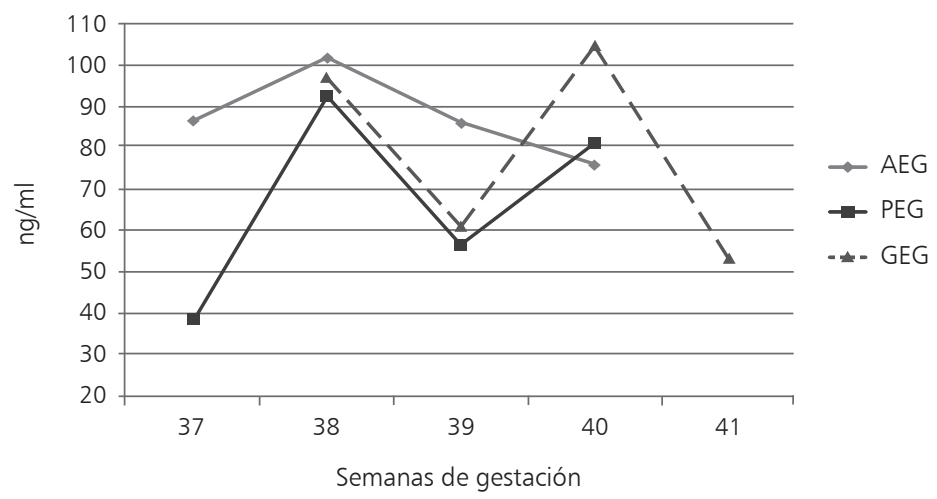

Figura 3. Valores en mediana de IGF-1, y edad gestacional pediátrica en RN de término. 


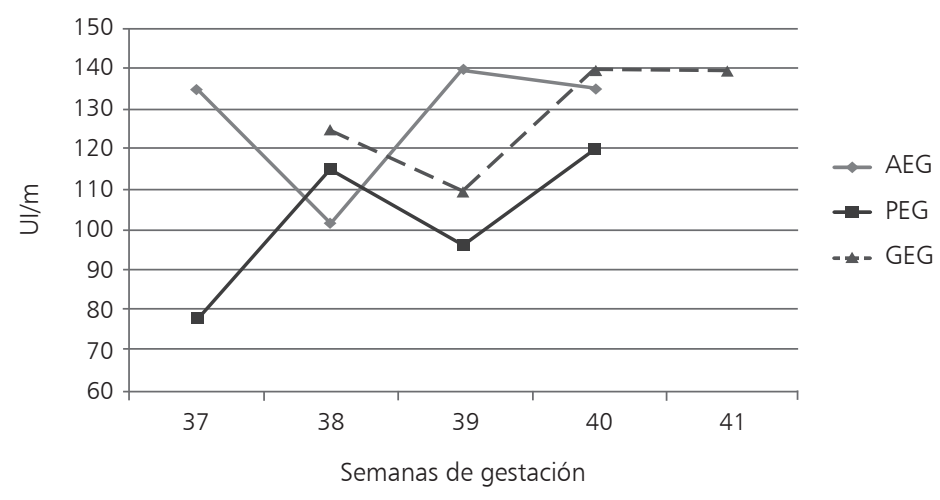

Figura 4. Valores de mediana de IGFBP-3 y edad gestacional pediátrica en $\mathrm{RN}$ de término.

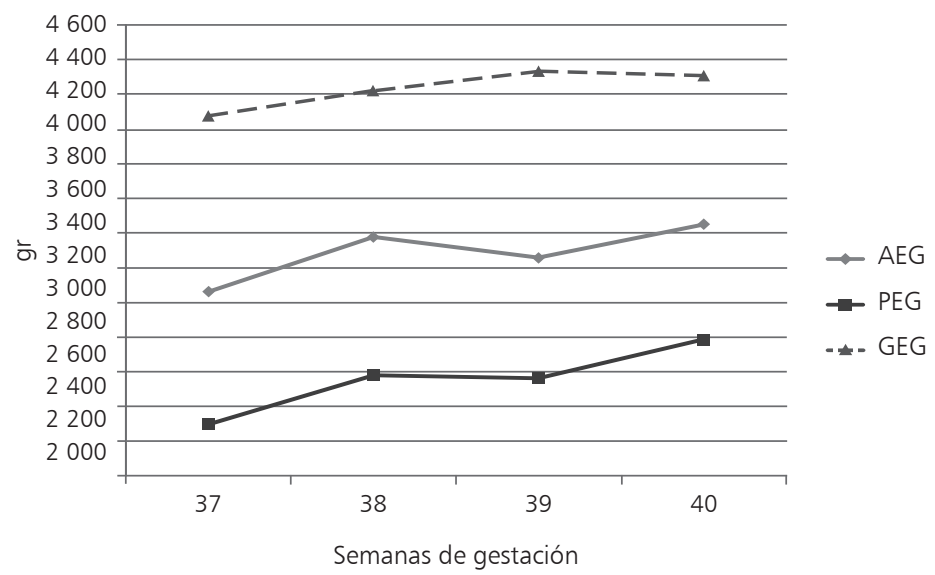

Figura 5. Peso promedio y edad gestacional pediátrica en RN de término.

\section{Discusión}

Los factores de crecimiento insulina, cortisol IGF-1 e IGFBP3 no mostraron diferencias significativas entre los recién nacidos RN AEG, PEG, y GEG a diferencia de lo reportado por la literatura. Sin embargo, al clasificar los recién nacidos por edad gestacional, se observa que las medianas de los factores de crecimiento en los diferentes grupos de $\mathrm{RN}$, son menores en los RN PEG y mayores en los RN AEG y GEG. Estos resultados pueden explicarse probablemente por el tamaño y elección de la muestra, la dificultad para la recolección de ésta, debido a la poca aceptación de las madres para participar en el estudio

La correlación positiva más evidente se observó entre la IGF-1 y el peso fetal en los tres grupos de RN. Este hallazgo concuerda con lo publicado por Eulises Díaz y col, quienes hicieron una revisión de la literatura con respecto al papel fisiológico del IGF-1 y sus proteínas transportadoras durante el embarazo, demostrando resultados similares ${ }^{15}$.

Al analizar los niveles de Insulina, IGF-1 e IGFBP-3 en el grupo PEG, se observa menores concentraciones que en los RN AEG y GEG respectivamente. Giudice y col, encontraron que los RN bajo condiciones nutricionales desfavorables, presentan menores concentraciones circulantes de IGF-1 e IGFBP-3 respecto de los $\mathrm{RN} \mathrm{AEG}^{16}$. A su vez Christou ${ }^{17}$, analizó los niveles de IGF-1, IGF-2, IGFBP-3, insulina y cortisol en suero de cordón umbilical en $142 \mathrm{RN}$ de término; observó que en RN GEG los niveles IGF-1, IGF-2 e insulina fueron significativamente más altos que en RN PEG y que los niveles de IGFBP-3 y cortisol muestran diferencias respecto de los RN GEG pero no significativas entre ambos grupos, obteniendo los mismos resultados que en nuestro estudio.

Por otra parte, Grandi en una población urbana de Buenos Aires sobre 9613 casos en que relacionó la antropometría materna y la ganancia de peso gestacional con el peso de nacimiento, no encontró diferencias significativas entre la talla materna y la ganancia de peso durante la gestación; los resultados de este estudio concuerdan con lo obtenido en nuestra investigación, no encontrando diferencias en estas mismas variables ${ }^{18}$.

En un estudio realizado por Lagos R y col, en Temuco, obtuvo diferencias significativas en IMC y peso al inicio de la gestación relacionado con el peso de 
los recién nacidos, observando diferencias en el peso de los $\mathrm{RN}$, en relación al IMC materno; estos resultados concuerdan con los obtenidos en nuestro estudio especialmente en lo referido al IMC materno al inicio de la gestación ${ }^{19,20}$.

Un comentario especial merece la gráfica de la curva de peso de los RN clasificados por tamaño en la cual se observa que los RN PEG en promedio ganan el $21 \%$ de su peso desde las 37 a las 40 semanas, en cambio los RN AEG el $12 \%$ y los GEG el 5,5\%. Esta observación podría tener una importante significación clínica en cuanto a la decisión de la interrupción del embarazo antes de las 40 semanas. En un estudio previo realizado por este grupo se observó que la ganancia de peso promedio de los $\mathrm{RN}$ de las $37 \mathrm{a}$ las 40 semanas fue de $600 \mathrm{~g}$ de promedio ${ }^{21}$.

En conclusión, el presente estudio muestra que los niveles de factores de crecimiento IGF-1, IGFBP-3 y cortisol en sangre de cordón de RN AEG, PEG y GEG, no muestran diferencias estadísticamente significativas entre los grupos; sin embargo, los de los RN AEG y GEG duplican los de los RN PEG. Las variables antropométricas maternas que presentan una correlación positiva significativa con el peso del RN son peso al inicio y termino del embarazo e IMC al inicio de la gestación.

\section{Agradecimientos}

A la Sra. Maritza Flores Opazo, Directora del Dpto. de Salud Pública de la Facultad de Medicina de la Universidad de Concepción por su fundamental aporte y colaboración.

A las Matronas del Servicio de Obstetricia y Ginecología, Unidad de Partos del Hospital Guillermo Grant Benavente de Concepción y de la Clínica Francesa de Concepción por su colaboración en la recolección de muestras.

\section{Referencias}

1.- Murphy V, Smith R, Warwick B., Giles, Vicki C: Endocrine Regulation of Human Fetal Growth: The Role of the Mother, Placenta and Fetus. Endocrine Rev 2006; 27: 141-69.
2.- Styne D: Crecimiento fetal. Clin Perinatol 1998; 4: 96989.

3.- Wooding F, Forhead A, Constancia M: Programming placental nutrient transport capacity A. L. Fowden, J. W. Ward. J Physiol 2006; 572: 5-15.

4.- Hales $C N$, Barker DJ: The thrifty phenotype hypothesis. Br Med Bull 2001;60: 5-20.

5.- Verkauskiene $R$, Beltrand J, Claris $O$, et al: Impact of fetal growth restriction on body composition and hormonal status at birth in infants of small and appropriate weight for gestational age. Eur J Endocrinol 2007; 157 : 605-12.

6.- Fowden A, Forhead A: Endocrine mechanisms of intrauterine programming. Reproduction 2004; 127: 515-26.

7.- Soto N, Mericq V: Restricción del crecimiento fetal e insulinorresistencia. Nuevos hallazgos y revisión de la literatura. Rev Med Chile 2005; 133: 97-104.

8.- Díaz E, Cárdenas M, Ariza A, Larrea F, Halhali A: Placental insulin and insulin-like growth factor I receptors in normal and preeclamptic pregnancies. Clin Biochem 2005; 38: 243-7.

9.- Carrascosa A: Crecimiento intrauterino: factores reguladores. Retraso de crecimiento intrauterino. An Pediatr 2003; 58: 55-73.

10.- Struwe E, Berzl GM, Schild RL, et al: Simultaneously reduced gene expression of cortisol-activating and cortisol-inactivating enzymes in placentas of small-forgestational-age neonates. Am J Obstet Gynecol 2007; 197: 43-6.

11.- Ellman L, Dunkel Ch, Calvin J, Chicz-Demet A, Glynn L, Sandman C: Timing of Fetal Exposure to Stress Hormones: Effects on Newborn Physical and Neuromuscular Maturation. Dev Psychobiol 2008; 50: 232-41.

12.- Tibblin G, Ericksson M, Cnattingius S, Ekbom A: High birth weight as a predictor of prostate cancer risk. Epidemiology 1995; 6: 423-4.

13.- Michels K, Trichopoulos D, Robins JM, et al: Birth weight as a risk factor for breast cancer. Lancet 1996; 348: 1542-6.

14.- Potter JD: Colorectal cancer: molecules and populations. J Natl Cancer Inst (Bethesda) 1999; 91: 916-32.

15.- Díaz E, Pichardo-Bahena R, Larrea F, Halhali A: Papel fisiológico del factor de crecimiento similar a la insulina tipo 1 y sus proteínas transportadoras durante el embarazo. Médica Sur, México MG 2004; 11: 91-8.

16.- Geudice L: Maternal-fetal conflict-lesson from a transgene. J Clin Invest 2002; 110: 307-9.

17.- Christou H, Connors J, Ziotopoulou M, et al: Cord 
blood leptin and insulin-like growth factor levels are independent predictors of fetal growth. J Clin Endocrinol Metab 2001; 86: 935-8.

18.- Grandi $C$ : Relación entre la antropometría materna y la ganancia de peso gestacional con el peso de nacimiento y riesgo de bajo peso al nacer, pequeños para la edad gestacional y premature en una población urbana de Buenos Aires. Arch Latinoam Nutric Venezuela 2003; 53: $\mathrm{N}^{\circ} 4$.

19.- Lagos R, Espinoza R, Orellana J: Estado nutritivo materno inicial y peso promedio de sus recién nacidos a término. Rev Chi Nutr 2004, 31: 52-7.

20.- Lagos R, Espinoza R, Orellana J: Antropometría maternal y peso promedio de nacimiento. Rev Chil Obstet Ginecol 2001; 2: 99-103.

21.- Cabrera J, Cruz G, Cabrera C, Cisterna M, Soto C, Sepulveda K, et al: Características del peso, edad gestacional, tipo de parto de recién nacidos en el Sistema Público y Privado. Rev Chil Obstet Ginecol 2006; 71: 92-7. 